\title{
Investigation of the garnet-perovskite transition in Nd doped YIG by means of magnetic disaccommodation
}

\author{
P. Hernández-Gómez ${ }^{* 1}$, C. De Francisco ${ }^{1}$, C. Torres ${ }^{1}$, J. Iñiguez ${ }^{2}$, V. Raposo², \\ J. M. Perdigao ${ }^{3}$, and A. R. Ferreira ${ }^{3}$ \\ ${ }^{1}$ Dpt. Electricidad y Electrónica, Universidad de Valladolid, Prado de la Magdalena s/n, \\ 47071 Valladolid, Spain \\ ${ }^{2}$ Dpt. Física Aplicada, Universidad de Salamanca, Pza. la Merced s/n, 37071 Salamanca, Spain \\ ${ }^{3}$ Dpt. Engenharia Electrotécnica e Computadores, Universidade de Coimbra, Pinhal de Marrocos, \\ 3030 Coimbra. Portugal
}

Received 31 August 2003, accepted 31 December 2003

Published online 18 March 2004

PACS 75.50.Gg, 75.60.Lr, 81.40.Rs

The garnet-perovskite transition is studied in this work with magnetic disaccommodation on polycrystalline Nd doped YIG samples $\mathrm{Y}_{3-\mathrm{x}} \mathrm{Nd}_{\mathrm{x}} \mathrm{Fe}_{5} \mathrm{O}_{12}(0<\mathrm{x}<2.5)$. A very different behaviour with the sintering atmosphere is observed. The results of magnetic disaccommodation for samples sintered in air reveal that the low temperature peak of YIG at $120 \mathrm{~K}$ disappear and another peak emerge at $300 \mathrm{~K}$ with $\mathrm{x}=2.0$, when the solubility limit is surpassed and the compound contains secondary perovskite phase and magnetite. For $\mathrm{CO}_{2}$ sintered samples, the process at $120 \mathrm{~K}$ exhibit magnetic accommodation features (i.e. negative disaccommodation), and vanishes with increasing Nd substitution, together with the appearance of the $300 \mathrm{~K}$ peak associated with the garnet-perovskite transition which takes place with $\mathrm{x} \approx 1.4$ doping. Thermal evolution of magnetic permeability and X-ray diffraction support the behaviour observed.

(c) 2004 WILEY-VCH Verlag GmbH \& Co. KGaA, Weinheim

\section{Introduction}

The study of magnetic relaxations, which is technologically important to minimize losses, provides also information about the underlying mechanisms governing the dynamic behaviour of Bloch walls [1]. Measurement of magnetic disaccommodation is a sensitive way to study magnetic relaxations in ferrites. It consists in the time variation of the mobility of domain walls after a magnetic shock, and is shown by a temporal evolution of the magnetic permeability after a demagnetization stage. The origin of this relaxation phenomenon, which has been observed both in cubic spinel ferrites [1], hexaferrites [2] and garnets [3], has been attributed to either the rearrangement or the diffusion of anisotropic point defects (lattice vacancies, interstitials) within the Bloch walls [1], and the relaxation time which characterizes each relaxation process is strongly temperature-dependent. Yttrium iron garnets (YIG) are excellent ferrimagnetic materials for use in microwave devices due to their good properties - high electrical resistivity and narrow resonance linewidth - at high frequencies together with the possibility of tailor their properties by introducing dopants [4]. Usually the tailoring is made by substitution of cations in tetrahedral or octahedral sites. The substitution of part of yttrium ions in dodecahedral sites with a rare earth element has been studied so far [5-7], and it has been established that in the garnet lattice total substitution of Y by lighter 4f rare earth elements $\mathrm{La}, \mathrm{Ce}, \mathrm{Pr}$ and $\mathrm{Nd}$ is not possible, and that above the solubility limit a secondary phase perovskite forms together with the magnetic garnet. In this paper we investigate the garnetperovskite transition in Nd doped YIG by using magnetic disaccommodation techniques.

* Corresponding author: e-mail: pabloher@ee.uva.es, Phone: +34 983 423895, Fax: +34 983423225 


\section{Experimental setup}

For this work, polycrystalline garnet samples with different $\mathrm{Nd}$ doping rate $\mathrm{Y}_{3-\mathrm{x}} \mathrm{Nd}_{\mathrm{x}} \mathrm{Fe}_{5} \mathrm{O}_{12}(0<\mathrm{x}<2.5)$ have been prepared by means of standard ceramic techniques. The mixtures were prepared with high purity starting oxides $\mathrm{Fe}_{2} \mathrm{O}_{3}, \mathrm{Y}_{2} \mathrm{O}_{3}$ and $\mathrm{Nd}_{2} \mathrm{O}_{3}$, mixed with the appropriate molar ratio in agate mortar for $1 \mathrm{~h}$., pressed at $10000 \mathrm{Kg} / \mathrm{cm}^{2}$ in cylindrical form (diameter: $5 \mathrm{~mm}$, length: $15 \mathrm{~mm}$ ), and sintered at temperatures in the $1380{ }^{\circ} \mathrm{C}<\mathrm{T}<1420^{\circ} \mathrm{C}$ range in air or $\mathrm{CO}_{2}$ sintering atmospheres. The samples were rapidly quenched to avoid phase annealing and to provide the presence of crystal vacancies.

Magnetic disaccommodation measurements were carried out with a computer aided system based on a LCR bridge [8], in the $77 \mathrm{~K}<\mathrm{T}<400 \mathrm{~K}$ temperature range. The results have been represented as isochronal curves, i.e, the relative variation of the initial permeability after sample demagnetization between an initial time $t_{1}=2 \mathrm{~s}$ and different window times $t_{2}=4,8,16,32,64$ and $128 \mathrm{~s}$ in the form

$$
\frac{\mu\left(t_{1}, T\right)-\mu\left(t_{2}, T\right)}{\mu\left(t_{1}, T\right)}(\%) .
$$

When the time window $\left(t_{2}-t_{1}\right)$ is of the same order of magnitude that the relaxation time at a specified temperature, this curve exhibits a maximum. In this way, isochronal spectra disclose the different aftereffect processes in the temperature range tested.

\section{Results and discussion}

X-ray diffraction patterns of representative samples are shown in Fig. 1. Thermal evolution of magnetic permeability and isochronal disaccommodation spectra for air and $\mathrm{CO}_{2}$ sintered samples are represented in Figs. $2 \mathrm{a}$ and $2 \mathrm{~b}$, and $3 \mathrm{a}$ and $3 \mathrm{~b}$, respectively. The garnet-perovskite transition can be observed in all the experimental results. XRD for air sintered samples (Figs. 1a to 1c) reveals the appearance of additional lines, related to $\mathrm{NdFeO}_{3}$ distorted perovskite and magnetite, once reached the solubility limit, which in our case takes place with $\mathrm{x}=2.0$ doping rate. The calculated garnet lattice parameter $a$ obtained for our samples, increase for $12.405 \AA$ for $\mathrm{x}=0.6$ to $12.525 \AA$ for $\mathrm{x}=2$ and $12.529 \AA$ for $\mathrm{x}=2.4$, similar to the literature data [5,7], and which indicate the nearly lineal increase of unit cell with doping rate until the solubility limit is reached, which following [7] takes place with a substitution rate $\mathrm{x}=1.9$. The transition is also evident in the thermal evolution of magnetic permeability: in Fig. 2 a we can observe that $\mathrm{Nd}$ doped YIG behave in three different manners depending that the doping rate lies in the range $0<\mathrm{x}<1,1$ $<\mathrm{x}<2$ or $\mathrm{x}>2$. In the latter case the magnetic permeability falls down to a very low value, which is due to the formation of substantial amount of antiferromagnetic perovskite (see Fig. 1c). Finally, the isochronal disaccommodation spectra obtained for air sintered samples (Fig. 3a) show a low temperature disaccommodation process at around $120 \mathrm{~K}$ characteristic of yttrium magnetic garnets, called II peak in [3]. This peak is due to $\mathrm{Fe}^{3+}-\mathrm{Fe}^{2+}$ electronic hopping relaxation mechanism in octahedral sites of garnet lattice, and its peak temperature, and hence the activation energy of the relaxation process, decrease with the Nd-induced increase of lattice unit cell. The garnet-perovskite transition is observed by the strong decrease of this relaxation process, which finally disappears, due to the small amount of garnet formed compared with perovskite and magnetite. Another disaccommodation peak at room temperature $(300 \mathrm{~K})$ also reveals that the solubility limit has been reached with $\mathrm{x}=2.0$. This relaxation process is observed due to the presence of magnetite in the sample and their origin is caused by ionic reorientation of anisotropic ferrous cations in octahedral sites of spinel lattice.

In $\mathrm{CO}_{2}$ sintered YIG (Fig. 3b) we can observe accommodation processes (i.e. negative disaccommodation) at low temperatures. This fact is due to the existence of time dependent wall resonance effects in garnets, at frequencies close to that employed in our measurement device $(1 \mathrm{kHz})$ [9], so that the time evolution of magnetic permeability can increase after demagnetization and hence accommodation can be observed. The peak also shifts to lower temperatures, and the $300 \mathrm{~K}$ peak characteristic of the presence 
of magnetite in the samples, and hence indicating that the solubility limit has been reached, appears with $\mathrm{x}=1.6$, a lower doping rate than air sintered samples. For higher substitution rates the low temperature garnet-related process disappears and it can be observed only the room temperature peak, due to the great amount of perovskite and magnetite formed. The thermal dependence of magnetic permeability (Fig. 2b) support this conclusion. We observe also three steps, depending that $\mathrm{x}<1.6, \mathrm{x}=1.6$ or $\mathrm{x}\rangle 1.6$, indicating, respectively, pure garnet formation, onset of secondary perovskite and magnetite formation, and substantial amount of perovskite and magnetite in the sample (and hence strong diminution of magnetic permeability). XRD obtained for the $\mathrm{CO}_{2}$ sintered sample with $\mathrm{x}=1.6$ doping rate (Fig. 1c) confirms that the solubility limit has been surpassed, as the intensities of $\mathrm{NdFeO}_{3}$ perovskite are comparable to that the corresponding of YIG (whose lattice parameter calculated is $a=12.465 \AA$ ). Taking into account the value for air atmosphere [7] and comparing the relative intensities of Figs. 1b and 1d, the most probable value for this limit in $\mathrm{CO}_{2}$ is $\mathrm{x} \approx 1.4$, but additional samples are needed to check it. We can conclude that the solubility limit in this system decreases with reducing atmospheres, probably due to a higher oxygen vacancy amount [9] that favours secondary phases formation. This fact also opens the possibility of increase the $\mathrm{Nd}$ doping rate by increasing oxygen partial pressure, for which further research is neccesary.

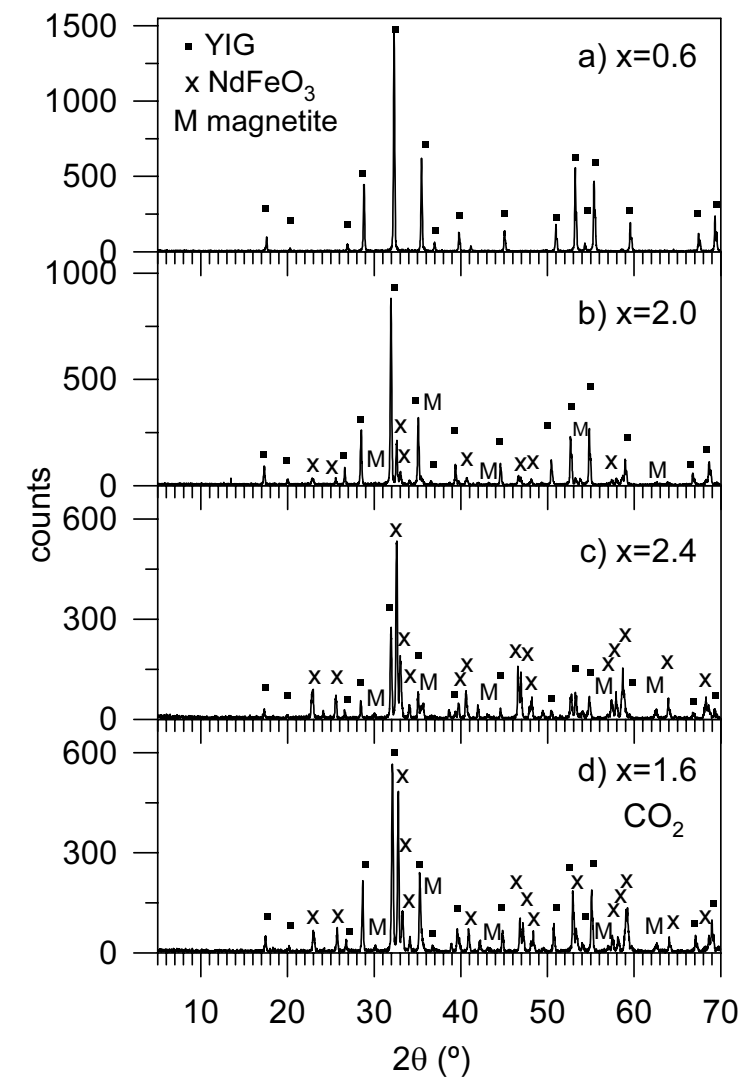

Fig. 1 XRD patterns of $\mathrm{Y}_{3-\mathrm{x}} \mathrm{Nd}_{\mathrm{x}} \mathrm{Fe}_{5} \mathrm{O}_{12}$ polycrystalline samples a) $\mathrm{x}=0.6, \mathrm{~b}) \mathrm{x}=2.0, \mathrm{c}) \mathrm{x}=2.4$ and d) $\mathrm{x}=1.6$ (sintered in $\mathrm{CO}_{2}$ ).[?: YIG; $\mathrm{x}: \mathrm{NdFeO}_{3}$; $\mathrm{M}$ : magnetite].

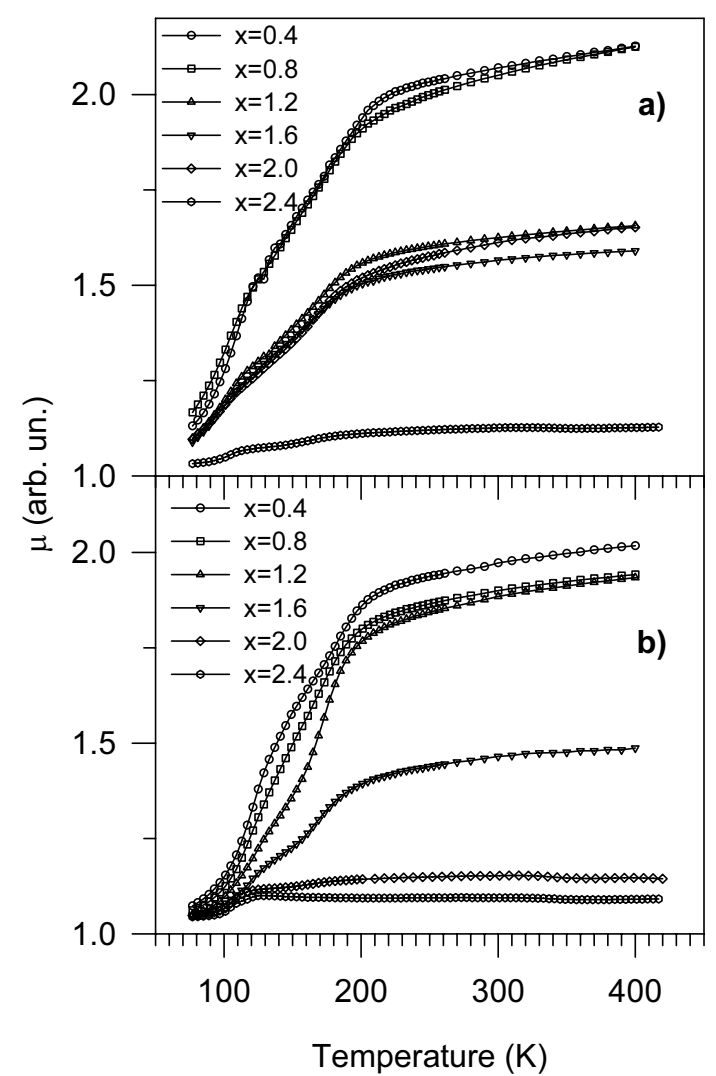

Fig. 2 Thermal evolution of magnetic permeability of $\mathrm{Y}_{3-\mathrm{x}} \mathrm{Nd}_{\mathrm{x}} \mathrm{Fe}_{5} \mathrm{O}_{12}$ polycrystalline samples sintered in a) air and b) $\mathrm{CO}_{2}$. 

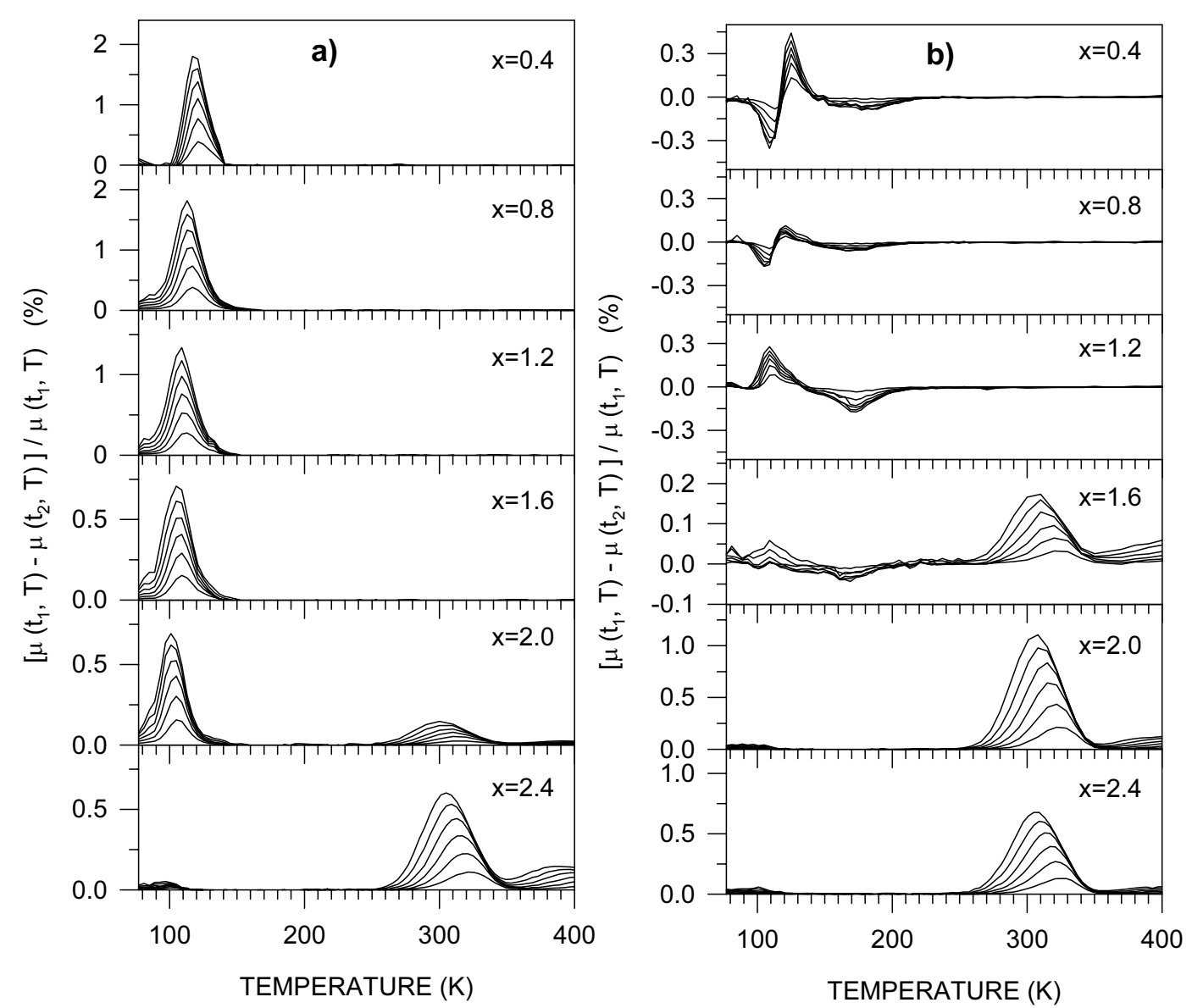

Fig. 3 Isochronal disaccommodation spectra of $\mathrm{Y}_{3-\mathrm{x}} \mathrm{Nd}_{\mathrm{x}} \mathrm{Fe}_{5} \mathrm{O}_{12}$ polycrystalline samples sintered in a) air and b) $\mathrm{CO}_{2}$ sintering atmospheres.

Acknowledgements This work has been partially supported by MCYT “Acciones Integradas Hispano-Lusas" and Junta de Castilla y León, project numbers HP2002-0014 and SA/010/03 resp.

\section{References}

[1] F.Walz, V.A.M. Brabers, S. Chikazumi, H. Kronmuller, and M.O. Rigo, phys. stat. sol. (b) 110, 471Q (1982).

[2] P. Hernández-Gómez, P.G. Bercoff, C. de Francisco, J.M. Muñoz, O. Alejos, C. Torres, and H.R. Bertorello, J. Appl. Phys. 87(9), 6250 (2000).

[3] L. Torres, F.Walz, J. Iñiguez, and H. Kronmüller, phys. stat. sol. (a) 159, 485 (1997).

[4] M. Pardavi-Horvath, J. Magn. Magn. Mater. 215-216, 171 (2000).

[5] J. Loriers and G. Villiers, Compt. Rend. Acad. Sci. 252, 1590 (1961).

[6] H. Pascard, J. Magn. Magn. Mater. 15-18, 1313 (1980).

[7] S. Geller, H.J. Williams, and R.C. Sherwood, Phys. Rev. 123(5), 1692 (1961).

[8] C. de Francisco, J. Iñiguez, J.M. Muñoz, and J. Ayala, IEEE Trans. Mag. 23, 1866 (1987).

[9] C. Torres, O. Alejos, J. M. Muñoz, P. Hernández-Gómez, and C. de Francisco, Phys. Rev. B 66, 024410 (2002). 